\title{
ESTUDIO SOBRE LOS SERVICIOS OFRECIDOS EN ECOLODGES PARA EL SECTOR ECONÓMICO ALTO DE LIMA METROPOLITANA
}

\author{
STUDY ON THE SERVICES OFFERED BY ECOLODGES FOR THE SECTOR ECONOMIC HIGH OF METROPOLITAN \\ LIMA.
}

https://doi.org/10.52109/cyp2021114

\author{
Leslie Alvariño ${ }^{1}$, Ayme Tapia ${ }^{1}$, Rafael Ramírez ${ }^{2}$ \\ ${ }^{1}$ Facultad de Administración y Recursos Humanos. Universidad de San Martin de Porres \\ 2 Doctor, Profesor Principal de la Universidad Nacional Agraria La Molina - ORCID: 0000-0003-3000-7089
}

REGISTROS

Recibido el 15/08/2020

Aceptado el 30/09/2020

Publicado el 31/01/2021

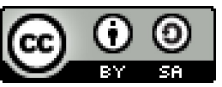

\section{PALABRAS CLAVE \\ Bionegocios, \\ ecoturismo, \\ estudios de mercado.}

\section{KEYWORDS}

Bio business, ecotourism, market studies.

\section{RESUMEN}

En los últimos años, el cuidado del ambiente y la gestión ambiental han preocupado a gobiernos, naciones y empresas, debido a que el inadecuado uso de los recursos naturales ha ido mermando la sostenibilidad del ambiente. Ante esta problemática y nuevo interés en cuidar y proteger el ambiente, nacen negocios enfocados hacia esta nueva tendencia; dentro de ellos los hoteles ecológicos o ecolodges. Por ese motivo el objetivo de esta investigación fue determinar el efecto de la preocupación ambiental en la intención de compra de los potenciales consumidores de hoteles ecológicos en Lima Metropolitana. Se utilizó el método descriptivo simple a través de una encuesta que permitió y determinar la intención de compra, la tendencia y la inclinación por los ecolodges, además del efecto e importancia que tiene la preocupación ambiental para la elección de estos por encima de los hoteles convencionales. Se identificó la presencia de la preocupación ambiental y su efecto positivo en la intención de compra de los potenciales consumidores de ecolodges en Lima Metropolitana. Por tanto, se concluyó que la preocupación ambiental es un factor importante para orientar al consumidor en elegir ecolodges durante sus viajes al interior del país.

\footnotetext{
ABSTRACT

In recent years, environmental care and environmental management have been a concern for governments, nations and many companies, because inadequate use of natural resources has been diminishing the sustainability of the environment. Faced with this problematic and with new interest in caring for and protecting the environment, businesses that focus on this new trend have been born; eco-friendly hotels or ecolodges. For this reason, the objective of this research was to determine the effect of environmental concern of potential consumers to purchase eco-friendly hotels in Metropolitan Lima. The simple descriptive method was used through a survey that allowed and determined the intent to buy, the trend and inclination for ecolodges, in addition to the effect and importance of environmental concern for the choice of these over conventional hotels. Se identified the presence of environmental concern and its positive effect on the intention of ecolodge consumers in Metropolitan Lima. It was therefore concluded that environmental concern is an important factor in guiding consumers in choosing ecolodges during their journeys to the interior of the country.
} 


\section{INTRODUCCIÓN}

En la actualidad la preocupación por el ambiente va aumentando, por ello son muchas las personas que ahora tratan de consumir productos y servicios que sean amigables con el ambiente y que buscan su cuidado.

El rubro hotelero no se encuentra excepto a esta tendencia ambiental, por ello nacen los ecolodges con el objetivo de tener una oferta hotelera dirigida a los viajeros y vacacionistas cada vez más conscientes de los problemas ambientales.

Como antecedente importante, tenemos lo señalado en el artículo $3^{\circ}$ del Decreto Supremo N029-2004-MINCETUR - Reglamento de Establecimientos de Hospedajes, que dividía a los establecimientos de hospedaje en las siguientes categorías:

Hotel: aquel establecimiento que cuenta con no menos de 20 habitaciones y que ocupa la totalidad de un edificio o parte de este completamente independizado, constituyendo sus dependencias una estructura homogénea. Los establecimientos de hospedaje para ser categorizados como Hoteles de 1 a 5 estrellas.

Apart-Hotel: Establecimiento de hospedaje que está compuesto por departamentos que integran una unidad de explotación y administración. Los Apart-Hoteles pueden ser categorizados de 3 a 5 estrellas.

Hostal: Establecimiento de hospedaje que cuenta con no menos de 6 habitaciones y que ocupa la totalidad de un edificio o parte del mismo completamente independizado, constituyendo sus dependencias una estructura homogénea. Los establecimientos de hospedaje para ser categorizados como Hostales de 1 a 3 estrellas.

Resort: Establecimiento de hospedaje ubicado en zonas vacacionales, tales como playas, ríos y otros de entorno natural, que ocupa la totalidad de un conjunto de edificaciones y posee una extensión de áreas libres alrededor del mismo. Los Resorts pueden ser categorizados de 3 a 5 estrellas.

Ecolodge: Establecimiento de hospedaje cuyas actividades se desarrollan en espacios naturales, cumpliendo los principios del Ecoturismo. Debe ser operado y administrado de una manera sensible, en armonía con el respeto y protección del medio ambiente.

Albergue: Establecimiento de hospedaje que presta servicio de alojamiento preferentemente en habitaciones comunes, a un determinado grupo de huéspedes que comparten uno o varios intereses y actividades afines, que determinarán la modalidad del mismo". (Artículo $3^{\circ}$ ).

Este fue un primer antecedente en el país para distinguir a esta clase de centros hoteleros y poder caracterizarlos, lo cual fue dejado sin efecto por el Decreto 
Supremo 001-2015-MINCETUR; sin embargo, su definición y diferencia con otras instalaciones sirve aun de referencia para caracterizar esta clase de locales de hospedaje, que incluso utilizan comercialmente esta definición.

El Perú tiene contados ecolodges, debido a que poco a poco se está desarrollando este nuevo rubro hotelero. Haciendo una revisión de diversos centros, se pudieron identificar a los siguientes como aquellos que cuentan con mayor número de características definidas comercialmente como "ecolodges":

Amantica Lodge, Casa Verde, Hotel Cabaña Quinta, Inkaterra La Casona, Killawasi Lodge Colca Perú, La Torre Valsai, Palmera de Asia, Puesta de Sol, Tradicion Colca, Wasai Puerto Maldonado Lodge y Wayqey Lodge (Amantica Lodge, 2017; Casa Verde, 2017; Hotel Cabaña Quinta, 2017; Inkaterra La Casona, 2017; Killawasi Lodge Colca Perú, 2017; La Torre Valsai,2017; Palmera de Asia, 2017; Puesta de Sol, 2017; Tradición Colca, 2017).

En cuanto a los precios que cobran algunos de estos establecimientos, se presentan en la tabla n. ${ }^{\circ}$ :

Tabla 1

Lista de precios de ecolodges

\begin{tabular}{lcccc}
\hline \multicolumn{1}{c}{ Ecolodge } & \multicolumn{4}{c}{ Precios (En Soles) } \\
& Simple & Doble & Triple & Promedio \\
\hline La Torre Valsai & 115.00 & 131.00 & 164.00 & 136.67 \\
La Quinta Ecohotel & 300.00 & 360.00 & 450.00 & 370.00 \\
Killawasi Lodge Colca Perú & 351.00 & 378.00 & 469.00 & 399.33 \\
Hotel Ecológico - Casa Verde & 189.00 & 189.00 & 236.00 & 204.67 \\
\hline Nota: fuente, Casa Verde, 2017; Hotel Cabaña Quinta, 2017; Killawasi Lodge Colca Perú, 2017; La Torre Valsai, 2017.
\end{tabular}

\section{La Teoría de la Acción Razonada}

Según Reyes, "la teoría de la acción razonada es una teoría general de la conducta humana que trata de la relación entre creencias, actitudes, intenciones y comportamiento, los cuales se encuentran relacionados con la toma de decisiones a nivel conductual" (Reyes, 2007. p 69), y cuyos elementos se aprecian en la figura $N^{\circ} 1$ siguiente: 
Figura 1

Elementos de la teoría de la acción razonada

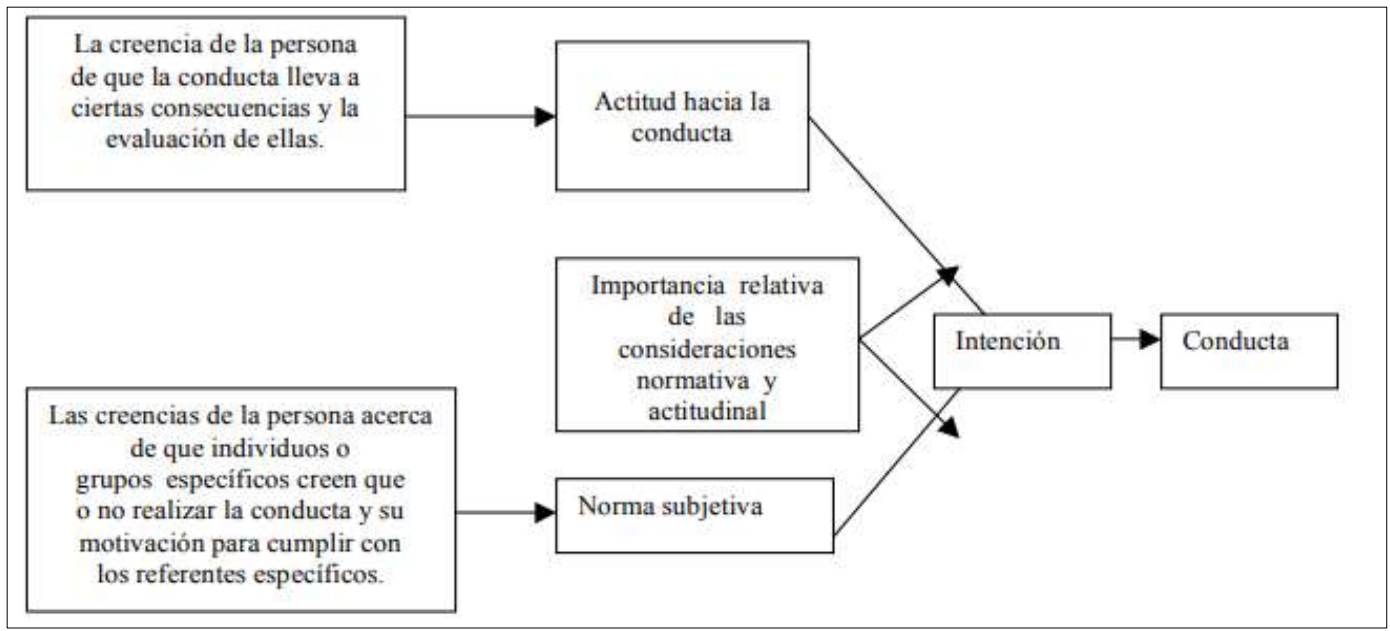

Nota: fuente, Reyes. L, 2007

Cabe resaltar que (Reyes, 2007) identifica ahí, los principales factores subjetivos que conforman la intención de compra y orientan la conducta humana hacia una decisión específica, aproximándose a la búsqueda de un indicador sobre la probabilidad en que una persona tome una decisión o asuma una conducta en base a estos factores.

\section{La intención de compra}

Sobre este aspecto, (Torres \& Padilla, 2013) definen la intención de compra como aquella decisión futura vinculada al comportamiento de un determinado consumidor.

Agrega (Marketing Directo, 2017) que la intención de compra es básicamente una preferencia especial por un servicio o bien, prefiriéndolo por sobre otras alternativas.

\section{Objetivo general del estudio}

Determinar la intención que presentan los potenciales consumidores de los Sectores "A" de Lima Metropolitana para alojarse en un ecolodge y su disponibilidad a pagar.

\section{MATERIALES Y MÉTODOS}

Dado que no se han encontrado estudios previos sobre el tema, el presente estudio utilizó un diseño descriptivo simple exploratorio a través de un muestreo probabilístico sobre 45 personas provenientes del universo formado por potenciales consumidores de los ecolodges pertenecerían al sector " $A$ " de los distritos de San Isidro, Miraflores y San Borja, que cumplieron con el siguiente perfil: Hombre o mujer mayor o igual de 30 años y menor a 50 años, con hijos, con ingreso 
mínimo mensual de S/. 5,000.00 soles, que viajen por vacaciones frecuentemente y que nunca hayan estado en un ecolodge.

Se empleó un cuestionario como instrumento de recolección de datos al que previo a su aplicación, se explicó a cada encuestado las características de cada tipo de alojamiento mostrándole fotos ilustrativas de condiciones estándar; los resultados se tabularon para su posterior sistematización, análisis y discusión.

\section{RESULTADOS}

Los resultados obtenidos se aprecian en las tablas $n .^{\circ} 2, n .^{\circ} 3, n .^{\circ} 4$ y n. ${ }^{\circ} 5$ siguientes:

Tabla 2

Disposición a alojarse en un ecolodge al momento de viajar independientemente del costo.

\begin{tabular}{lrr}
\hline \multicolumn{1}{c}{ Opinión } & Porcentaje & \multicolumn{2}{c}{ Porcentaje acumulado } \\
\hline Muy en desacuerdo & 2.20 & 2.20 \\
En desacuerdo & 11.10 & 13.30 \\
Indiferente & 33.30 & 46.70 \\
De acuerdo & 48.90 & 95.60 \\
Muy de acuerdo & 4.40 & 100.00 \\
Total & 100.00 & 100.00 \\
\hline
\end{tabular}

Tabla 3

Actitud frente al esfuerzo económico para alojarse en un ecolodge comparado al pago de un hotel convencional de igual nivel.

\begin{tabular}{lrr}
\hline \multicolumn{1}{c}{ Opinión } & Porcentaje & \multicolumn{2}{c}{ Porcentaje acumulado } \\
\hline Muy en desacuerdo & 0.00 & 0.00 \\
En desacuerdo & 8.90 & 8.90 \\
Indiferente & 26.70 & 35.60 \\
De acuerdo & 60.00 & 95.60 \\
Muy de acuerdo & 4.40 & 100.00 \\
Total & 100.00 & 100.00 \\
\hline
\end{tabular}


Tabla 4

Preocupación acerca del estado del ambiente en el Perú y en general en el mundo.

\begin{tabular}{|c|c|c|}
\hline Opinión & Porcentaje & Porcentaje acumulado \\
\hline No tiene ninguna & 0.00 & 0.00 \\
\hline Tiene alguna & 2.20 & 2.20 \\
\hline Indiferente & 11.10 & 13.30 \\
\hline Preocupado & 44.40 & 57.70 \\
\hline Muy preocupado & 42.30 & 100.00 \\
\hline Total & 100.00 & 100.00 \\
\hline
\end{tabular}

Tabla 5

Disponibilidad a pagar para alojarse en ecolodges (en porcentaje)

\begin{tabular}{ccccc}
\hline $\begin{array}{c}\text { Pago por noche de } \\
\text { alojamiento }(\mathrm{S} / .)\end{array}$ & $\begin{array}{c}\text { Excelente } \\
\text { precio }\end{array}$ & $\begin{array}{c}\text { Precio } \\
\text { razonable }\end{array}$ & $\begin{array}{c}\text { Quizás pagaría, } \\
\text { quizás no }\end{array}$ & $\begin{array}{c}\text { No pagaría de } \\
\text { ninguna manera }\end{array}$ \\
\hline 200.00 & 40.00 & 42.20 & 11.10 & 6.70 \\
300.00 & 15.60 & 53.30 & 17.80 & 13.30 \\
400.00 & 0.00 & 42.20 & 33.40 & 24.40 \\
700.00 & 0.00 & 0.00 & 24.40 & 75.60 \\
1000.00 & 0.00 & 0.00 & 6.70 & 93.30 \\
\hline
\end{tabular}

\section{DISCUSIÓN}

De la tabla $n .^{\circ} 2$, se desprende que, en general e independientemente del precio a pagar, por la solo condición de ser un hotel amigable con el ambiente, un 53.3\% de encuestados se halla de acuerdo o muy de acuerdo en alojarse en un ecolodge.

Este resultado es coherente con la actitud frente a alojarse en un hotel convencional, ya el $64.4 \%$ de la tabla $n .^{\circ} 3$ está de acuerdo o muy de acuerdo en preferir alojarse en un ecolodge que en un hotel convencional. Resulta interesante ver que cuando la pregunta involucra una comparación, el porcentaje sube de $53.3 \%$ a $64.0 \%$, lo cual refleja que los ecolodges tendrían un alto interés en estos potenciales clientes.

Durante la evaluación acerca de la preocupación ambiental por parte de la población evaluada, un $86.6 \%$ manifestó su preocupación por el estado general del ambiente, vale decir que en su mayoría son conscientes de los problemas que actualmente se viven vinculados al deterioro ambiental. Esta información resulta relevante, ya que concuerda con las hipótesis de otros estudios sociales que parte de considerar que los niveles socio económicos más elevados cuentan con una mayor conciencia ambiental. Sin embargo, como se aprecia en la Tabla n. ${ }^{\circ} 4$, existe 
un $13.30 \%$ de encuestados que son indiferentes o no tienen ninguna preocupación por la problemática ambiental; más allá de los fines del presente estudio, aún falta trabajar por mejorar la conciencia ambiental entre la población.

Finalmente, en la tabla $n .^{\circ} 5$ se presentan los resultados vinculados con la disponibilidad a pagar por alojarse en un ecolodge, el cual se determinó en base a aproximaciones sucesivas con diversos precios planteados por noche de alojamiento.

Los resultados nos arrojan, que aun cuando existe a priori una buena predisposición por utilizar este tipo de alojamiento, lo cual es alentador; no es menos cierto que el precio es el factor que permite cuantificar esta buena disposición.

En tal sentido, se puede apreciar que la disponibilidad a pagar general se haya entre los S/. 300.00 a S/. 400.00 por noche, este valor es el equivalente al precio cobrado por hoteles convencionales de 3 a 4 estrellas, lo que permite poder concretar con un valor, la valoración que los consumidores le dan a los ecolodges.

\section{CONCLUSIONES}

Se concluye que los sectores económicos más altos en Lima Metropolitana cuentan con una conciencia ambiental importante y que ello y la preocupación por el estado del ambiente son factores claves en la intención que lleva a su conducta de elegir un ecolodge como alternativa de alojamiento en viajes de turismo.

La disponibilidad a pagar por alojarse en un ecolodge se haya dentro de los precios ofrecidos por instalaciones hoteleras dentro del rango del mercado actual, en tal sentido esta alternativa se haya en adecuadas condiciones para competir con instalaciones convencionales de 3 a 4 estrellas.

La presente investigación es una contribución inicial sobre el tema, que permite tener un panorama alentador para interesados en invertir en implementar ecolodges y que quizás no toman esa decisión por no contar con la información disponible; en todo caso la recomendación es en base a este estudio prospectivo, desarrollar evaluaciones más específicas en otros sectores socioeconómicos nacionales o en turistas extranjeros.

Se recomienda que las empresas dedicadas al rubro hotelero tomen en cuenta la presente investigación y la importancia del cuidado ambiental, por ello es importante dirigir este sector a la perspectiva ecológica, debido a que existe demanda en este sector y podría ser considerado como un nuevo nicho de mercado o un mercado en crecimiento, realizado el análisis adecuado.

Es necesario que los gobiernos locales, regionales y el gobierno nacional concierten acciones y afirmen recursos para implementar políticas de apoyo al sector hotelero en lo que respecta a investigación, el fortalecimiento de políticas ambientales y 
brindar una asistencia de orientación hacia el cuidado del ambiente de forma ágil y oportuna.

\section{REFERENCIAS}

Amantica Lodge. (2017). Amantica Lodge. Consultado el 10 de enero del 2017 http://amanticalodge.com/experiencia.html

Casa Verde. (2017). Casa Verde Ecolodge. Consultado el 13 enero 2017 http://www.casaverde.pe/

Hotel Cabaña Quinta. (2017). Hotel Cabaña Quinta. Consultado el 10 enero 2017 https://www.booking.com/hotel/pe/cabana-quinta.es.html

Inkaterra (2017). Inkaterra La Casona. Consultado el 13 enero 2017 http://www.inkaterra.com

Killawasi Lodge Colca Perú. (2017). Killawasi Lodge. Consultado el 12 de enero del 2017 http://www.killawasilodge.com/es/

La Torre Valsai. (2017). La Torre Valsai. Consultado el 12 enero 2017 https://www.booking.com/hotel/pe/la-torre-valsai.es.html

Marketing Directo (2017). Intención de Compra. Consultado el 14 marzo 2017 https://www.marketingdirecto.com/diccionario-marketing-publicidadcomunicacion-nuevas-tecnologias/intencion-de-compra-2

Palmera de Asia. (2017). Palmera de Asia. Consultado el 12 enero 2017 https://www.booking.com/hotel/pe/las-palmeras-de-asia.es.html

Ministerio de Comercio Exterior y Turismo. (2004, 27 de noviembre). Decreto Supremo 029-2004-MINCETUR. Diario oficial El Peruano Año XXI- 8927, p. 281121-281135.

Ministerio de Comercio Exterior y Turismo. (2015, 9 de junio). Decreto Supremo 001 2015-MINCETUR. Diario oficial El Peruano Año XXXII-13286, p. 554647554655.

Puesta de Sol. (2017). Puesta de Sol. Consultado el 12 enero 2017 https://www.booking.com/hotel/pe/puesta-de-sol.es.html

Reyes, L. (2007). La Teoría de la Acción Razonada. Implicaciones para el estudio de las actitudes.

Investigación Educativa Duranguense. №. 7, 2007, págs. 66-77. ISSN-e 2007-039X. https://dialnet.unirioja.es/servlet/articulo?codigo=2358919

Torres, E.; Padilla, G. (2013). Medición de la intención de compra con base en un modelo de regresión logística de productos de consumo masivo. [tesis de pregrado, Universidad Politécnica Salesiana de Ecuador]. Disponible en: http://dspace.ups.edu.ec/handle/123456789/5772

Tradicion Colca. (2017). Tradicion Colca. Consultado el 12 enero 2017 https://www.booking.com/hotel/pe/tradicion-colca.es.html

Wasai Puerto Maldonado Lodge. (2017). Wasai Puerto Maldonado Lodge. Consultado el 12 enero 2017 https://www.booking.com/hotel/pe/wasaipuerto-maldonado-eco-lodge.es.html

Wayqey Lodge. (2017). Wayqey Lodge. Consultado el 12 enero 2017 https://www.booking.com/hotel/pe/wayqey-lodge.es.html 